\title{
Knowledge, perception and implementation of personal protective measures by citizens during the COVID-19 outbreak in Northern Cyprus: a cross- sectional survey
}

\section{Azze Buket Yilmaz}

Department of Pharmaceutical Microbiology, Faculty of Pharmacy, Near East University, Nicosia, Cyprus Emrah Guler

Department of Medical Microbiology and Clinical Microbiology, Faculty of Medicine, Near East University, Nicosia, Cyprus

Buket Baddal ( $\sim$ buket.baddal@neu.edu.tr)

Department of Medical Microbiology and Clinical Microbiology, Faculty of Medicine, Near East University, Nicosia, Cyprus

\section{Research Article}

Keywords: COVID-19, personal protective equipment, personal protective measures, citizens, Northern Cyprus

Posted Date: July 21st, 2020

DOI: https://doi.org/10.21203/rs.3.rs-45269/v1

License: (c) (i) This work is licensed under a Creative Commons Attribution 4.0 International License. Read Full License

Version of Record: A version of this preprint was published at Cyprus Journal of Medical Sciences on November 1st, 2021. See the published version at https://doi.org/10.5152/cjms.2021.3086. 


\section{Abstract}

Aim: Severe acute respiratory syndrome coronavirus 2 (SARS-CoV-2) has generated over 8000000 cases of the coronavirus disease 2019 (COVID-19) worldwide and has led to the implementation of strict measures in all countries. The aim of this study was to investigate the knowledge, attitude and implementation status of personal protective measures (PPMs) by ordinary citizens in Northern Cyprus and their access to personal protective equipment (PPE) during the COVID-19 outbreak.

Subject and Methods: This was a cross-sectional study based on internet-based survey. A total of 406 participants were recruited between May 1 and 4, 2020 via authors' networks to complete a questionnaire. Participants were asked to indicate how often they implemented the five PPMs recommended by the World Health Organization (WHO), their daily frequency of hand hygiene events, their COVID-19 knowledge as well as the availability of PPE in their hometown.

Results: The prevalence of the five PPMs ranged from $65.0 \%$ to $95.6 \%$, with the highest being hand hygiene and the lowest being avoiding touching the eyes, nose, and mouth. Hand hygiene events were recorded to be more than 10 per day in $47.3 \%$ of the participants. Gloves (86.2\%), surgical masks $(52.2 \%)$, and cloth face masks (47.3\%) were the most commonly used PPE in public areas. The majority of the responders were aware of the incubation time $(90.4 \%)$ and indirect route of viral transmission $(89.7 \%)$, whereas knowledge of droplet (72.2\%) and aerosol transmission (43.6\%) were lower. Knowledge was gained mainly through social media and TV.

Conclusion: Overall, citizens implemented protective measures effectively and were strictly coherent to government-induced curfew and self-isolation measurements with a high public awareness.

\section{Introduction}

Originated from Wuhan, China in late 2019, severe acute respiratory syndrome coronavirus 2 (SARS-CoV2), the etiological agent of the coronavirus disease 2019 (COVID-19), has emerged as a significant threat to public health, and has been declared as a pandemic by the World Health Organization (WHO), with 8 061550 cases and 440290 deaths occurring worldwide as of $17^{\text {th }}$ June 2020 (World Health Organization a. 2020). While SARS-CoV-2 was initially observed in East Asia, Europe consequently became the center of the pandemic (World Health Organization b. 2020), followed by United States and Latin America, and is currently adversely affecting multiple African countries.

With the rapid surge in the number of people infected with SARS-CoV-2, WHO emphasized the urgent need to coordinate international collaborative efforts to minimize the threat in all affected countries in order to prevent the rapid spread of COVID-19 (World Health Organization c. 2020). While the battle against COVID-19 is ongoing, multiple measures have been taken by governments in an effort to slow down and mitigate viral transmission of the respiratory virus SARS-CoV-2 in the local population such as 
closing down schools, banning public gatherings/events, stopping mass movements via land, sea and airports, imposing partial/full curfew, implementation of the social distance rules and compulsory use of face masks. When formulating a health policy, key front-line workers within multi-disciplinary teams should be a part of the policy planning to ensure that these policies function effectively (Park et al. 2015). Indeed, government health education messages represent a key source of information for citizens for promoting their self-protective practices against respiratory infectious diseases. These preventive messages generally emphasize improved hygiene, face-mask use and social distancing measures, including avoiding crowds during epidemics (World Health Organization. 2009; Centers for Disease Control and Prevention. 2010; European Centre for Disease Prevention and Control. 2020). Although the decision-making and application of such measures by governmental bodies is essential for the control of a pandemic, the level of awareness and adherence of citizens to control measures is also crucial for the successful application of the aforementioned measures.

In the absence of an effective vaccine, public implementation of PPMs is fundamental. WHO has recommended five main PPMs against COVID-19: hand hygiene, social distancing measures, avoiding touching the eyes, nose and mouth, practice of respiratory hygiene and self-isolation (World Health Organization d. 2020). Another recommendation by WHO also suggests that citizens should follow advice given by their healthcare provider or their national and local public health authorities in order to obtain information from a reliable source. The level of public adherence to control measures is affected by their knowledge, attitudes, and practices towards COVID-19 and it directly impacts the trajectory of the outbreak in a country. This is particularly valid during outbreaks which, due to their evolving nature and inherent scientific uncertainties, can be associated with considerable fear in the general public, especially if illness or death rates are high (Person et al. 2004). This was a phenomenon observed during the SARS outbreak in 2003, where studies conducted suggest that the level of panic emotions in the population is directly associated with their knowledge and attitudes towards the infectious disease (Hung 2003).

In order to facilitate outbreak management of COVID-19 in Northern Cyprus, there is an urgent need to clarify the public's awareness of COVID-19 and their status of implementation of the protective measures, as well as their access to personal protective equipment (PPE) during the COVID-19 pandemic. The aim of this study is to investigate the knowledge, attitude and practices of Cypriot citizens towards COVID-19, and measure their coherence to PPMs during the public health crisis.

\section{Subjects And Methods}

Participants and data collection 
This was a cross-sectional study conducted through an internet-based survey between $1^{\text {st }}$ May and $4^{\text {th }}$ May, 2020. The survey ended on $4^{\text {th }}$ May 2020, which corresponds to the date on which COVID-19 related partial curfew from 06:00 to 21:00 ended in Northern Cyprus, in order to have reliable data on selfisolation and coherence to curfew measurements. As of $1^{\text {st }}$ May, the total number of reported COVID-19 cases in Northern Cyprus was 108 (Turkish Republic of Northern Cyprus Ministry of Health 2020). A total of 406 citizens were included in this study. Participants were recruited via authors' networks with local citizens living in Northern Cyprus. A recruitment poster with a link to questionnaire was posted to online pages with local COVID-19 related media as well as Facebook, emails and WhatsApp, which contained information on the background, objective, voluntary nature of participation, indications how to fill in the questionnaire as well as the declarations of anonymity and confidentiality of participants. No ethical approval was required for this survey.

\section{Measurement}

Assessment of sociodemographic characteristics

The first part of the questionnaire consisted of the assessment of sociodemographic attributes in which participants responded to demographic variables including gender, age, education status, occupation, current district of residency, smoking (smoker/non-smoker), chronic disease (present/non-present) and seasonal influenza vaccination history (vaccinated/non-vaccinated).

\section{Assessment of knowledge on COVID-19}

Participants were asked to answer questions regarding the transmission routes of SARS-CoV-2 among choices including droplet, aerosol and indirect transmission (touching contaminated surfaces and then touching eyes, mouth and nose) routes or indicate if they did not know the transmission route of infection. Participants also responded to a question on the incubation period for SARS-CoV-2. Among the 
choices 1-2 days, 2-4 days, 2-14 days, 27 days and "I do not know the incubation period" option were present.

Assessment of WHO recommended personal protective control measures

Participants were asked to indicate which of the personal protective control measures including hand hygiene, social distance, avoiding touching nose, mouth and eyes, and use of face mask they implemented during the pandemic and the frequency of implementation for each measure. For each option, the scale was designed as always, sometimes, rarely and never. Participants also responded to a question which assessed their coherence to the curfew imposed by the local government, outside of their working hours, with a scale of always, generally, rarely, and never.

The application of personal protective equipment by ordinary citizens was evaluated. Individuals were asked to indicate which PPE they used in public areas such as supermarkets, with choices including gloves, surgical masks, vented masks, cloth masks, wrap/scarf, face shield, goggles, and "I do not use any PPE". Participants were also assessed if they applied hand hygiene before and after PPE use, and if they adhered to social-distancing rules while wearing face masks.

Assessment of hand hygiene events per day

Questions were designed to evaluate the hand hygiene measures applied by citizens. Individuals were initially asked which products they use when they apply hand hygiene and selected from various options including water, soap and water, alcohol-based hand sanitizer, pure alcohol, cologne with at least $70 \%$ alcohol content, wet tissues or "I do not use any products". The number of hand hygiene events per day was also evaluated. Participants reported the mean number hand hygiene events based on soap and water and alcohol-based hand sanitizers per day.

Assessment of the availability of personal protective equipment during the pandemic 
As the use of PPE and application of hand sanitation can be affected by their availability in a local town or city, participants were asked to report their access to these products during the pandemic. Participants responded to questions for the availability of masks, gloves and hand sanitizers with a scale of always, generally, rarely and never for each product.

Assessment of the attitude towards healthcare facilities during the pandemic

The citizens were asked if, during the pandemic, they felt any reservation from visiting hospitals or any healthcare facility where they responded as yes or no. Additionally, participants were asked if they would go for a COVID-19 test in a mobile test center in a hospital carpark without leaving their car for which they responded as yes or no.

Assessment on the source of COVID-19 related information

In this survey, the use of reliable source of personal protective procedures as well as the source of COVID19 related updates by participants were evaluated. Individuals were asked to choose among World Health Organization, Turkish Republic of Northern Cyprus Ministry of Health, Turkish Republic of Northern Cyprus Medical Association, newspapers, TV and social media for the most commonly used source of personal protective procedures. Citizens also indicated the most commonly used sources of COVID-19 related updates among scientific articles, TV, radio, internet sites, newspapers, brochures/flyers, social media, family/friends/relatives and WhatsApp group.

Statistical analysis

Statistical analyses were performed using IBM SPSS Statistics for Windows, version 23 (IBM, Japan, Tokyo, Japan). Knowledge and attitudes and practices of different individuals according to demographic characteristics were compared with independent samples $t$ test or Chi-square test as appropriate. Binary linear regression analysis using demographic variables as independent variables and knowledge or 
practices as the outcome variable was conducted to identify factors associated with attitudes and practices. $P$ value 0.05 or less was considered significant.

\section{Results}

A total of 406 participants completed the survey questionnaire. The demographic characteristics of participants are shown in Table 1 . Of all the responders, $47 \%$ were males and $53 \%$ were females. The distribution of age range was $2 \%$ in the $15-20$ age group, $37.2 \%$ in the $21-34$ age group, $24.4 \%$ in $35-44$ age group, $9.4 \%$ in $45-54$ age group, $21.2 \%$ in the $55-64$ age group and $5.9 \%$ in the $65+$ age group. The highest number of participants were residents of Nicosia (51.5\%), followed by Kyrenia (17.5\%), Famagusta (15.5\%), Lefka (6.4\%), Morphou (4.9\%) and Trikomo (3.9\%). A large majority of the participants had a Bachelor's degree (49.8\%) or higher (24.9\%), while $17.7 \%$ had high school, $5.4 \%$ had secondary school and $2.2 \%$ had primary school education. Of all the responders, $71.4 \%$ were nonsmokers, while $28.6 \%$ were smokers. In terms of chronic disease, $16.7 \%$ answered 'Yes' and $83.3 \%$ answered ' $N o$ ', whereas only $6.4 \%$ of the individuals received the seasonal flu vaccination.

Before assessment of the implementation of PPMs by citizens, participants were asked two questions regarding the transmission routes and incubation time of COVID-19 in order to measure their level of knowledge of the disease, which may also affect their inclination to properly apply protective measures and PPE use. A striking $89.7 \%$ of the individuals responded with indirect transmission route for the disease, whereas $72.2 \%$ and $43.6 \%$ were aware of droplet transmission and aerosol transmission routes, respectively (Fig. 1a). A small proportion, $1.2 \%$ of the citizens responded "I do not know" for the disease transmission question. Regarding the incubation time for COVID-19, 90.4\% was aware of the 2-14 days of incubation time, and the rest of the individuals either answered "I do not know" or responded with a wrong choice of incubation time (Fig. 1b). Binary logistic regression analysis showed that education levels of individuals and COVID-19 incubation time knowledge were not significantly associated $(P=0.917)$, suggesting that individuals at all education levels were knowledgeable about COVID-19 and that this knowledge was gained through educatory channels during the pandemic.

Fig. 2a shows the prevalence of the application of WHO recommended PPMs against COVID-19 by the citizens. In terms of hand hygiene, $96.5 \%$ of the participants responded to always implement the measure during the pandemic. Social distancing was always implemented by $82.6 \%$ of the responders, while $15.6 \%$ and $1.8 \%$ responded to sometimes and rarely apply social distancing measures in public areas, respectively. Alternatively, $72.6 \%$ and $65.8 \%$ always implemented the use of face masks and avoided touching the nose, mouth and eyes, respectively. Among all PPMs, hand washing was statistically the most applied measure $(P=0.001)$. In terms of PPE use, \%86.2 of the citizens responded to use gloves, $2.0 \%$ to use googles, and $0.7 \%$ to use face shield while in public areas such as supermarkets. When face masks are considered, \%52.2 responded to use surgical masks, $47.3 \%$ to use cloth masks, $7.6 \%$ to use 
vented masks and $2.0 \%$ to use wrap or scarf to cover their mouth and nose while in public areas. Types of PPE used by participants and their usage prevalence is shown in Fig. $2 \mathrm{~b}$.

Of all the participants, $90.1 \%$ indicated to apply hand hygiene before and after mask and glove use, and $93.8 \%$ indicated to practice 2-meter social distancing while in public while using masks. Citizens were also asked how often they adhered to curfew imposed by the local government. A large proportion, 79.5\% were always and $19.5 \%$ were usually coherent to the curfew, whereas a total of $1 \%$ of the individuals rarely or never adhered to the imposed curfew (Table 2).

When participants were asked to indicate which hand hygiene products they used, $95.6 \%$ responded to use soap and water, $81 \%$ to use hand sanitizer, $\% 69$ to use cologne with at least $70 \%$ alcohol content, $38.4 \%$ to use absolute alcohol and $37.4 \%$ to use wet wipes. However, $4.7 \%$ used only water and none of the responders indicated not to use any hand hygiene product (Fig. 3a). Fig. 3b and c show the total number of hand hygiene events per day. For hand washing, the majority of participants, $47.3 \%$ washed their hands more than 10 times a day, followed by 7-10 times with $33.5 \%$, 4-6 times with $16.3 \%$ and $1-3$ times with $2.7 \%$ (Fig. 3b). For hand sanitation events, the majority of citizens, $37.9 \%$, applied hand sanitizer 1-3 times a day, followed by $4-6$ times by $28.8 \%, 7-10$ times by $13.5 \%$ and $8.6 \%$ of the participants never applied hand sanitizer (Fig. 3c). There was a statistically significant difference between age groups and hand washing frequency $(P=0.046)$, where individuals in age group 35-54 had a higher hand washing frequency (7 or more) compared with other groups. On the contrary, there was no statistically significant difference between age groups and frequency of hand sanitation application $(P=0.159)$.

When the local availability of PPE for the participants was assessed, only $20.5 \%, 26.8 \%$ and $26.8 \%$ responded to always have access to masks, gloves and hand sanitizer available, respectively. A large majority of participants responded to usually find masks (49.5\%), gloves (52.0\%), and hand sanitizer $(51.2 \%)$ available. However, although less than $25 \%$, some of the participants were rarely or never able to find these PPE products available at the pharmacies or supermarkets during the pandemic (Fig. 4).

Majority of citizens ( $82 \%$ ) indicated that, during the pandemic, they felt reservation from visiting hospitals or healthcare facilities. Additionally, \%84.2 of the participants responded that they would be comfortable with going for a COVID-19 test in a mobile test center in a hospital carpark without leaving their car. Binary logistic regression analysis showed that education levels of individuals and having a COVID-19 test in a mobile test center were not significantly associated $(P=0.266)$, suggesting that individuals at all education levels were comfortable with having a COVID-19 test in a mobile test center. 
Based on the responses as the source of COVID-19 related information, $71.4 \%$ of the participants indicated to use social media, $70.9 \%$ to use TV and $68.5 \%$ to use internet websites. Less than $20 \%$ of the citizens used newspapers, scientific articles, brochures/flyers, radio, family/friends/relatives and WhatsApp groups as source of COVID-19 information. Among the responders, the majority followed personal protective procedures via social media (73.6\%), TV (55.2\%) or newspapers (5.2\%), while the reliable sources such as World Health Organization, T.R.N.C Ministry of Health and T.R.N.C Medical Association ranged as $42.9 \%, 48.0 \%$ and $25.1 \%$, respectively (Table 3 ).

\section{Discussion}

In the course of a pandemic, the implementation of PPMs represents an important factor for the control of the outbreak and has been shown to have significant protective effects associated (Saunders-Hastings et al. 2017). This study was designed to assess the knowledge, attitudes and implementation status of PPMs by the citizens in Northern Cyprus between 1 May and 4 May 2020. The survey period covers an important time period amid COVID-19 pandemic in which all preventive measures enforced by the local government in Northern Cyprus was in place and the curfew was still ongoing, and therefore provides a timely assessment. Overall, $70-90 \%$ of the individuals surveyed were knowledgeable about the droplet and indirect transmission routes of disease transmission, whereas the citizens were aware of the more recently described aerosol route of transmission (van Doremalen et al. 2020) at a lesser extent, approximately $44 \%$. The survey responders also demonstrated a $90 \%$ knowledge of the incubation period for COVID-19, demonstrating a high comprehension of disease characteristics during the pandemic. Recent surveys on public knowledge on COVID-19 in China, where the pandemic has emerged, demonstrated an overall $90 \%$ correct rate on a COVID-19 knowledge test which included questions on main clinical symptoms, viral transmission, observation period and age groups affected (Zhong et al. 2020). In a separate study, where the responders were asked question regarding the origin, common signs and symptoms, sources of infection of COVID-19 and awareness of any other pandemic viral infection, the knowledge level of individuals varied according to profession (Khan et al. 2020).

WHO and its recommendation of five PPMs against COVID-19, namely hand hygiene, social distancing measures, avoiding touching the eyes, nose and mouth, use of face masks, and self-isolation, has been taken as a reference by all countries and was communicated to the citizens via local health authorities in each country. Of these measures, the prevalence of hand hygiene and social distancing were the highest 
and respiratory etiquette was high, whereas the prevalence of avoiding touching nose, mouth and eyes was the lowest among Northern Cyprus citizens. Compared with a recent a study conducted in Japan, with hand hygiene being the highest with $83.8 \%$ and the lowest being avoiding touching eyes, nose and mouth with 59.8\%, the prevalence of PPM application was overall higher in Northern Cyprus (Machida et al. 2020). Similarly, in a population survey performed in the United Kingdom in the age group of 18+ individuals, the prevalence of respondents taking the aforementioned measures to protect themselves and others from COVID-19 were comparably much lower with face mask implementation being as low as $3 \%$ and hand washing being highest with $83 \%$ (Atchison et al. 2020). Alternatively, in a study conducted in Hong Kong, enhanced personal hygiene practices were adopted by more than $77 \%$ of citizens.

The recommendation on the use of face masks by citizens in public areas has been controversial. In a guidance report dated 29 January 2020, the WHO recommendation was for only individuals with respiratory symptoms to wear a medical mask to avoid unnecessary cost, procurement burden and false sense of security (World Health Organization e. 2020). WHO, additionally published interim guidance report on the rational use of PPE for COVID-19 which recommended the use of vented masks (respirators) only for frontline healthcare workers in order to optimize the PPE availability (World Health Organization f. 2020). However, health authorities in parts of Asia encouraged all citizens to wear masks in public setting, and the Czech Republic applied mandatory use of face masks to prevent viral spread in the community (Servick 2020). The Centers for Disease Prevention and Control (CDC) has also recommended the use of cloth face coverings or cloth masks in public settings with particular risk of community-based transmission (Centers for Disease Prevention and Control. 2020). In Northern Cyprus, the mandatory use of face masks was implemented by the local government on $24^{\text {th }}$ April 2020. In the Cypriot community, the practice of surgical mask and cloths mask use was $47-52 \%$ and approximately $8 \%$ for vented masks, indicating a good adherence to government measures against COVID-19 with some room for improvement.

During the early phase of the pandemic, a major demand of face masks, gloves and hygiene products arose mainly due to the panic caused by the outbreak and it has led to the exhaustion of local hygiene products and personal protective equipment as well as a steep rise in prices in many countries (Chaib 2020). In Northern Cyprus, the shortage of gloves, face masks and hand sanitizers were reported by 21 $30 \%$ of the citizen, who indicated they could never find certain PPE types available. In terms of the source of information used by people to gather knowledge about COVID-19, some studies suggested that social media and internet is among the most commonly used sources (Abdelhafiz et al. 2020; Atchison et al. 2020), whereas people also had a tendency of acquiring information and advice from TV and local health authorities which they expressed as more reliable (Kwok et al. 2020). The Cypriot citizens' choice of COVID-19 information and advice on PPMs were similar to previous studies with TV, social media and 
local health authorities such as Ministry of Health and Medical Association being represented on top of the list.

In the course of a pandemic, the implementation of PPMs represents an important factor for the control of the outbreak and has been shown to have significant protective effects associated (Saunders-Hastings et al. 2017). This study was designed to assess the knowledge, attitudes and implementation status of PPMs by the citizens in Northern Cyprus between 1 May and 4 May 2020. The survey period covers an important time period amid COVID-19 pandemic in which all preventive measures enforced by the local government in Northern Cyprus was in place and the curfew was still ongoing, and therefore provides a timely assessment. Overall, $70-90 \%$ of the individuals surveyed were knowledgeable about the droplet and indirect transmission routes of disease transmission, whereas the citizens were aware of the more recently described aerosol route of transmission (van Doremalen et al. 2020) at a lesser extent, approximately $44 \%$. The survey responders also demonstrated a $90 \%$ knowledge of the incubation period for COVID-19, demonstrating a high comprehension of disease characteristics during the pandemic. Recent surveys on public knowledge on COVID-19 in China, where the pandemic has emerged, demonstrated an overall $90 \%$ correct rate on a COVID-19 knowledge test which included questions on main clinical symptoms, viral transmission, observation period and age groups affected (Zhong et al. 2020). In a separate study, where the responders were asked question regarding the origin, common signs and symptoms, sources of infection of COVID-19 and awareness of any other pandemic viral infection, the knowledge level of individuals varied according to profession (Khan et al. 2020).

WHO and its recommendation of five PPMs against COVID-19, namely hand hygiene, social distancing measures, avoiding touching the eyes, nose and mouth, use of face masks, and self-isolation, has been taken as a reference by all countries and was communicated to the citizens via local health authorities in each country. Of these measures, the prevalence of hand hygiene and social distancing were the highest and respiratory etiquette was high, whereas the prevalence of avoiding touching nose, mouth and eyes was the lowest among Northern Cyprus citizens. Compared with a recent a study conducted in Japan, with hand hygiene being the highest with $83.8 \%$ and the lowest being avoiding touching eyes, nose and mouth with 59.8\%, the prevalence of PPM application was overall higher in Northern Cyprus (Machida et al. 2020). Similarly, in a population survey performed in the United Kingdom in the age group of 18+ individuals, the prevalence of respondents taking the aforementioned measures to protect themselves and others from COVID-19 were comparably much lower with face mask implementation being as low as $3 \%$ and hand washing being highest with $83 \%$ (Atchison et al. 2020). Alternatively, in a study conducted in Hong Kong, enhanced personal hygiene practices were adopted by more than $77 \%$ of citizens. 
The recommendation on the use of face masks by citizens in public areas has been controversial. In a guidance report dated 29 January 2020, the WHO recommendation was for only individuals with respiratory symptoms to wear a medical mask to avoid unnecessary cost, procurement burden and false sense of security (World Health Organization e. 2020). WHO, additionally published interim guidance report on the rational use of PPE for COVID-19 which recommended the use of vented masks (respirators) only for frontline healthcare workers in order to optimize the PPE availability (World Health Organization f. 2020). However, health authorities in parts of Asia encouraged all citizens to wear masks in public setting, and the Czech Republic applied mandatory use of face masks to prevent viral spread in the community (Servick 2020). The Centers for Disease Prevention and Control (CDC) has also recommended the use of cloth face coverings or cloth masks in public settings with particular risk of community-based transmission (Centers for Disease Prevention and Control. 2020). In Northern Cyprus, the mandatory use of face masks was implemented by the local government on $24^{\text {th }}$ April 2020. In the Cypriot community, the practice of surgical mask and cloths mask use was $47-52 \%$ and approximately $8 \%$ for vented masks, indicating a good adherence to government measures against COVID-19 with some room for improvement.

During the early phase of the pandemic, a major demand of face masks, gloves and hygiene products arose mainly due to the panic caused by the outbreak and it has led to the exhaustion of local hygiene products and personal protective equipment as well as a steep rise in prices in many countries (Chaib 2020). In Northern Cyprus, the shortage of gloves, face masks and hand sanitizers were reported by 21$30 \%$ of the citizen, who indicated they could never find certain PPE types available. In terms of the source of information used by people to gather knowledge about COVID-19, some studies suggested that social media and internet is among the most commonly used sources (Abdelhafiz et al. 2020; Atchison et al. 2020), whereas people also had a tendency of acquiring information and advice from TV and local health authorities which they expressed as more reliable (Kwok et al. 2020). The Cypriot citizens' choice of COVID-19 information and advice on PPMs were similar to previous studies with TV, social media and local health authorities such as Ministry of Health and Medical Association being represented on top of the list.

\section{Conclusion}

In conclusion, the knowledge of the disease characteristics and the practice of PPMs and coherence to government induced protective measures was high in Cyprus citizens. This is believed to have advantageous effects on the control of the outbreak in the country where no new cases of COVID-19 has been reported since $17^{\text {th }}$ April 2020 (AA Media. 2020).

\section{References}


AA Media. (2020) Northern Cyprus reports no new COVID-19 cases in weeks

Abdelhafiz AS, Mohammed Z, Ibrahim ME, et al (2020) Knowledge, Perceptions, and Attitude of Egyptians Towards the Novel Coronavirus Disease (COVID-19). J Community Health.

https://doi.org/10.1007/s10900-020-00827-7

Atchison C, Bowman L, Eaton JW, et al (2020) Public Response to UK Government Recommendations on COVID-19: Population Survey, 17-18 March 2020. Imp Coll London COVID-19 Response Team

Centers for Disease Control and Prevention. (2010) CDC says "take 3" actions to fight the flu.

Centers for Disease Prevention and Control. (2020) Use of Cloth Face Coverings to Help Slow the Spread of COVID-19

Chaib F (2020) Shortage of personal protective equipment endangering health workers worldwide. In: Who

European Centre for Disease Prevention and Control. (2020) Personal protective measures (nonpharmaceutical) for reducing the risk of acquiring or transmitting human influenza

Hung LS (2003) The SARS Epidemic in Hong Kong: What Lessons have we Learned? J R Soc Med. https://doi.org/10.1177/014107680309600803

Khan S, Khan M, Maqsood K, et al (2020) Is Pakistan prepared for the COVID-19 epidemic? A questionnaire-based survey. J Med Virol. https://doi.org/10.1002/jmv.25814

Kwok KO, Li KK, Chan HH, et al (2020) Community responses during the early phase of the COVID-19 epidemic in Hong Kong: risk perception, information exposure and preventive measures. medRxiv. https://doi.org/10.1101/2020.02.26.20028217

Machida M, Nakamura I, Saito R, et al (2020) Adoption of personal protective measures by ordinary citizens during the COVID-19 outbreak in Japan. Int J Infect Dis. https://doi.org/10.1016/j.ijid.2020.04.014

Park Y-S, Lee C, Kim KM, et al (2015) The first case of the 2015 Korean Middle East Respiratory Syndrome outbreak. Epidemiol Health. https://doi.org/10.4178/epih/e2015049

Person B, Sy F, Holton K, et al (2004) Fear and Stigma: The Epidemic within the SARS Outbreak. Emerg. Infect. Dis.

Saunders-Hastings P, Crispo JAG, Sikora L, Krewski D (2017) Effectiveness of personal protective measures in reducing pandemic influenza transmission: A systematic review and meta-analysis. Epidemics 
Servick K (2020) Would everyone wearing face masks help us slow the pandemic? Science (80-). https://doi.org/10.1126/science.abb9371

Turkish Republic of Northern Cyprus Ministry of Health (2020) COVID-19 Announcement.

van Doremalen N, Bushmaker T, Morris DH, et al (2020) Aerosol and Surface Stability of SARS-CoV-2 as Compared with SARS-CoV-1. N Engl J Med. https://doi.org/10.1056/nejmc2004973

World Health Organization. (2009) Pandemic influenza prevention and mitigation in low resource communities.

World Health Organization a. (2020) Coronavirus disease (COVID-19) Situation Report.

World Health Organization b. (2020) WHO announces COVID-19 outbreak a pandemic

World Health Organization c. (2020) 2019-nCoV outbreak is an emergency of international concern

World Health Organization d. (2020) Coronavirus disease (COVID-19) advice for the public

World Health Organization e. (2020) Advice on the use of masks in the community, during home care and in healthcare settings in the context of the novel coronavirus (2019-nCoV) outbreak.

https://apps.who.int/iris/handle/10665/330987

World Health Organization f. (2020) Rational use of personal protective equipment for coronavirus disease 2019 ( COVID-19)

Zhong BL, Luo W, Li HM, et al (2020) Knowledge, attitudes, and practices towards COVID-19 among Chinese residents during the rapid rise period of the COVID-19 outbreak: a quick online cross-sectional survey. Int J Biol Sci. https://doi.org/10.7150/ijbs.45221

\section{Declarations}

Acknowledgements: We would like to thank all study participants who have provided us with their valuable information and time to accomplish the web-based survey.

Funding: This research did not receive financial support from any funding agencies. 


\section{Compliance with Ethical Standards}

Conflict of Interest: The authors declare that they have no known competing financial interests or personal relationships that could have appeared to influence the work reported in this paper.

Ethical Considerations: Respondent's anonymity and confidentiality were ensured. The submission of the answered survey was considered as consent to participate in the study.

\section{Tables}

Table 1. Demographic characteristics of participants 


\section{Participant characteristics}

Gender

Age group (years)

Residential area

Education

Smoking

Chronic disease
$15-20$

21-34

35-44

45-54

55-64

$65+$

Kyrenia

Nicosia

Famagusta

Trikomo

Morphou

Lefka

Primary school

Secondary school

High school

Bachelor's degree

Master's degree or above
Number of participants, $\mathbf{n}(\%)$

191 (47)

$215(53)$
8 (2)

$151(37.2)$

99 (24.4)

$38(9.4)$

86 (21.2)

$24(5.9)$
71 (17.5)

210 (51.7)

63 (15.5)

16 (3.9)

$20(4.9)$

$26(6.4)$

9 (2.2)

22 (5.4)

72 (17.7)

202 (49.8)

101 (24.9)

116 (28.6)

$290(71.4)$

Non-smoker

68 (16.7)

338 (83.3) 


$\begin{array}{lll}\text { Seasonal influenza vaccination } & \text { Yes } & 26(6.4) \\ & \text { No } & 380(93.6)\end{array}$

Table 2. Participant coherence to curfew imposed by the local government

Always $\mathrm{n} \quad$ Usually $\mathrm{n} \quad$ Rarely $\mathrm{n} \quad$ Never $\mathrm{n}$

(\%)

(\%)

$323(79.5) \quad 79(19.5)$

$(\%)$

(\%)

Coherence to curfew - self isolation measures

Table 3. The source of COVID-19 related information and personal protective measures 


\begin{tabular}{ll} 
Source of personal protective procedures & $\mathbf{n}(\%)$ \\
\hline Social Media & $299(73.6)$ \\
TV & $224(55.2)$ \\
T.R.N.C Ministry of Health & $195(48.0)$ \\
World Health Organization & $174(42.9)$ \\
T.R.N.C Medical Association & $102(25.1)$ \\
\hline Newspaper & $21(5.2)$ \\
\hline Source of COVID-19 information & $\mathbf{n}(\%)$ \\
\hline Social media & $290(71.4)$ \\
TV & $288(70.9)$ \\
Internet sites & $278(68.5)$ \\
Scientific articles & $83(20.4)$ \\
Newspaper & $40(9.9)$ \\
Family/friends/relatives & $40(9.9)$ \\
WhatsApp group & $23(5.7)$ \\
Radio & $16(3.9)$ \\
\hline Brochures/flyers & $4(1)$
\end{tabular}


Figures

A

B
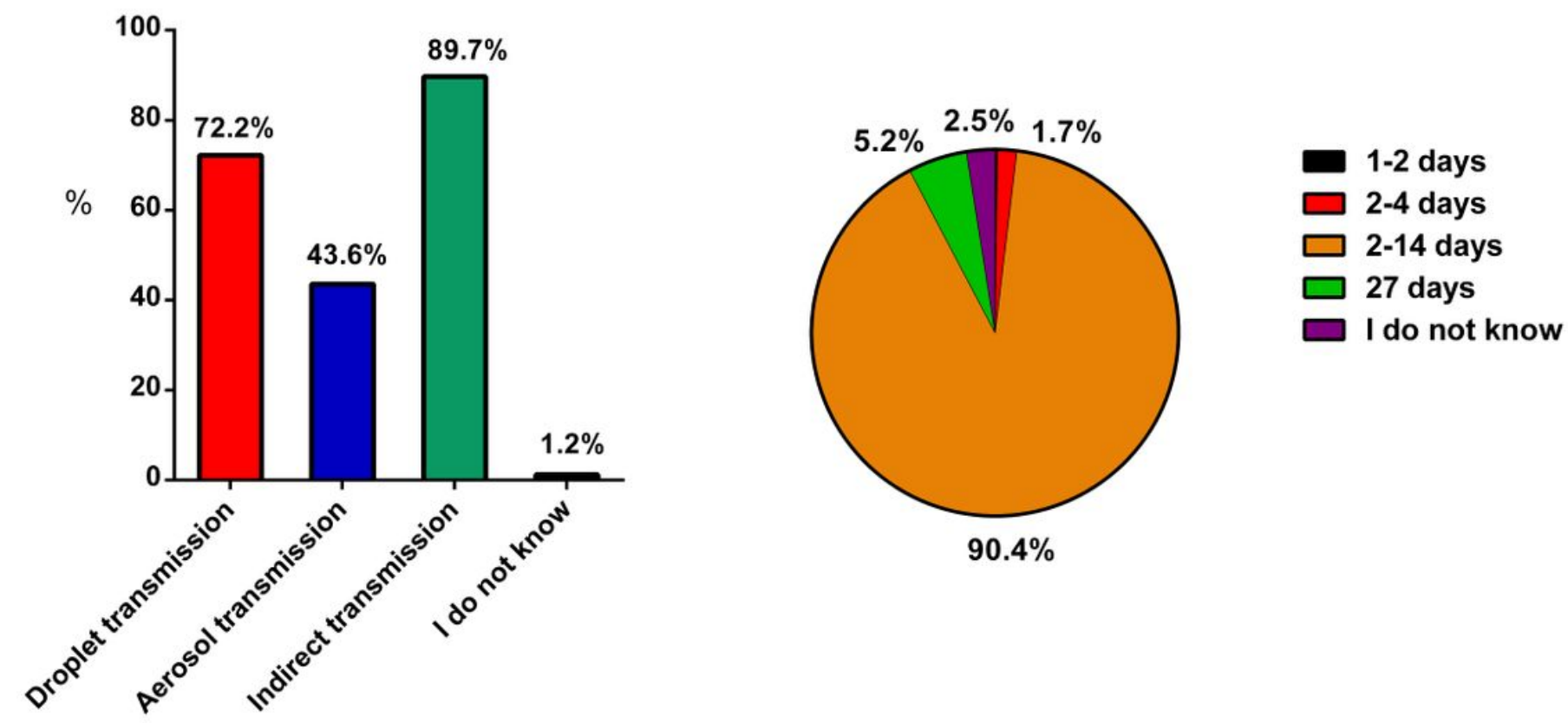

Figure 1

Participant knowledge on COVID-19 characteristics 
A

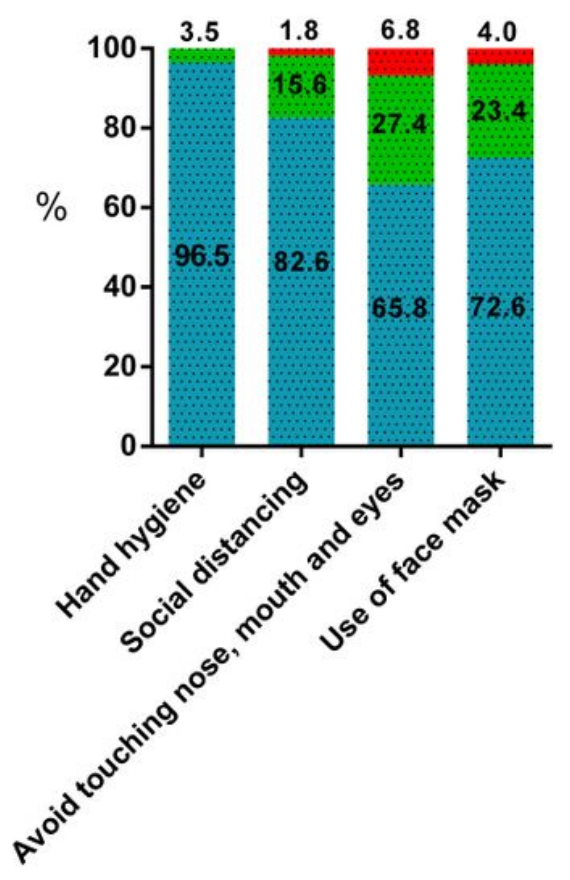

B

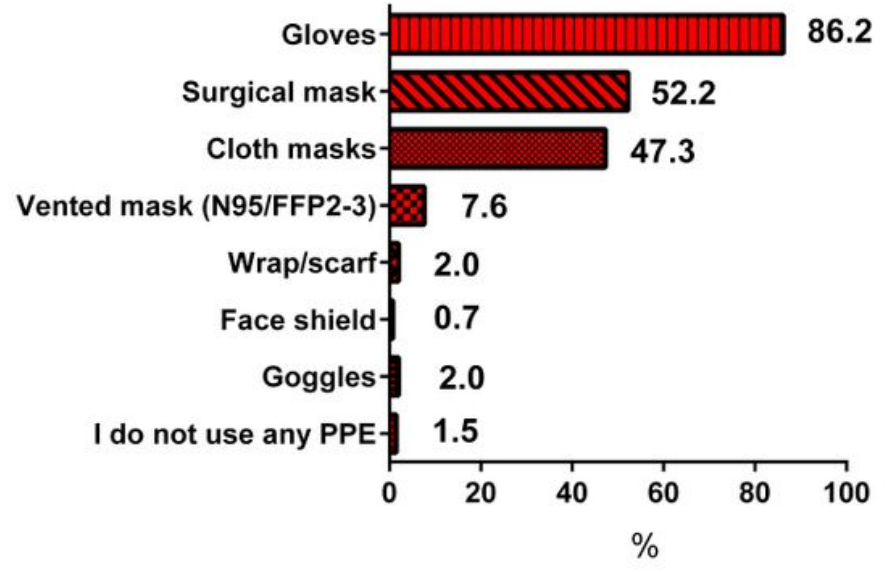

Figure 2

Application frequency of personal protective measures recommended by $\mathrm{WHO}$ and use of personal protective equipment by participants 
A

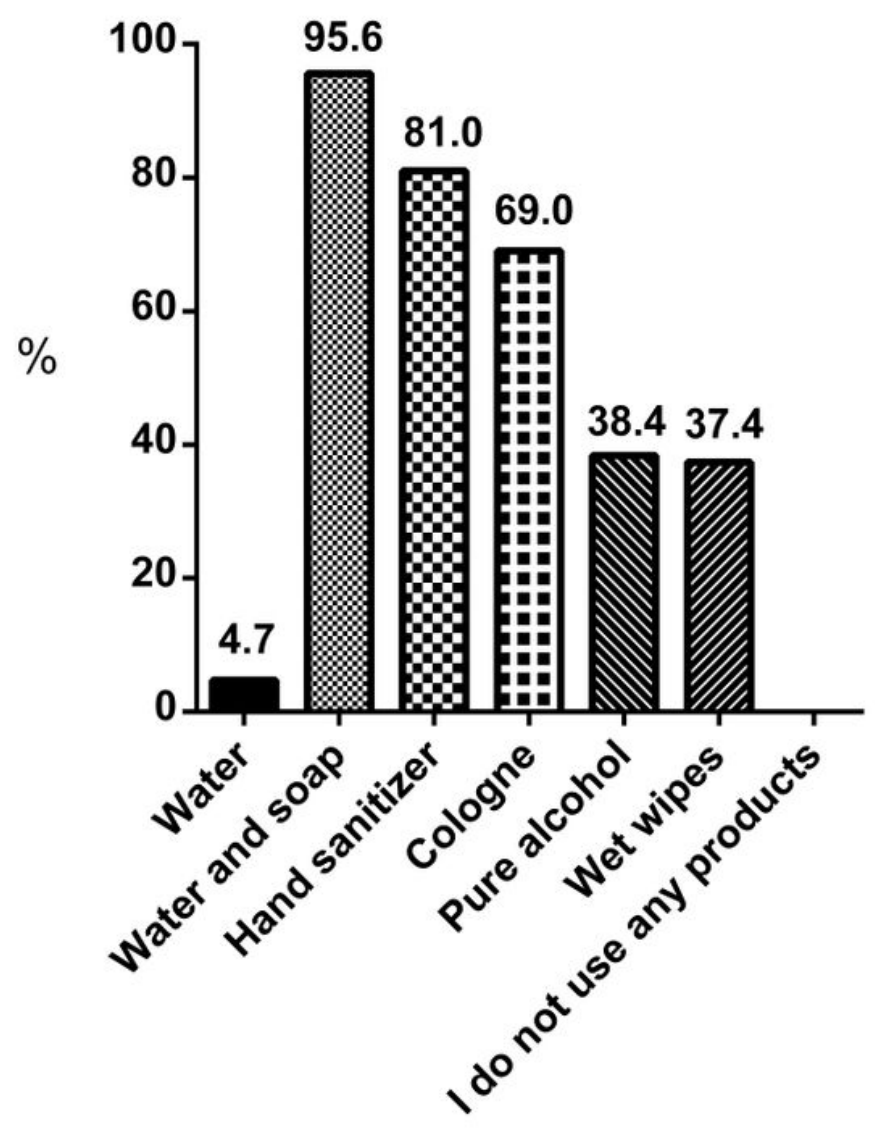

B
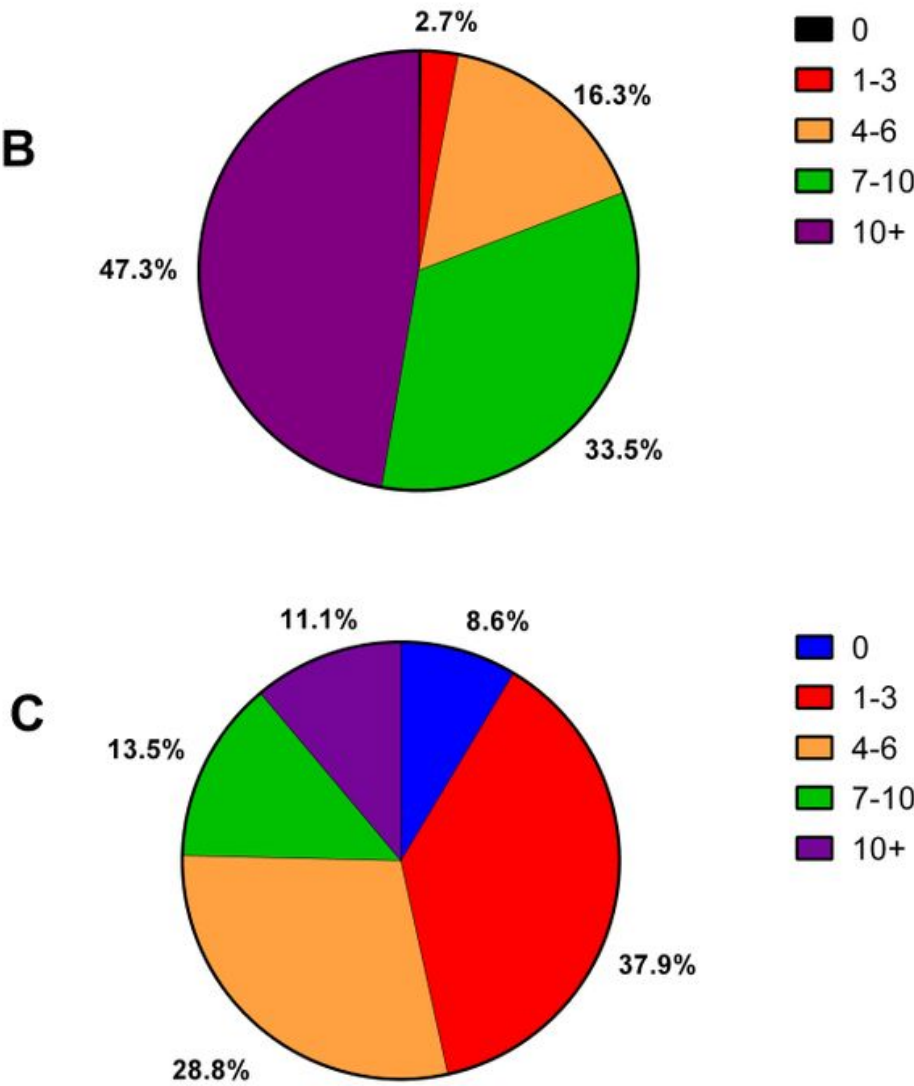

Figure 3

The proportion of participants applying hand hygiene products and frequency of daily hand washing and hand sanitation events 


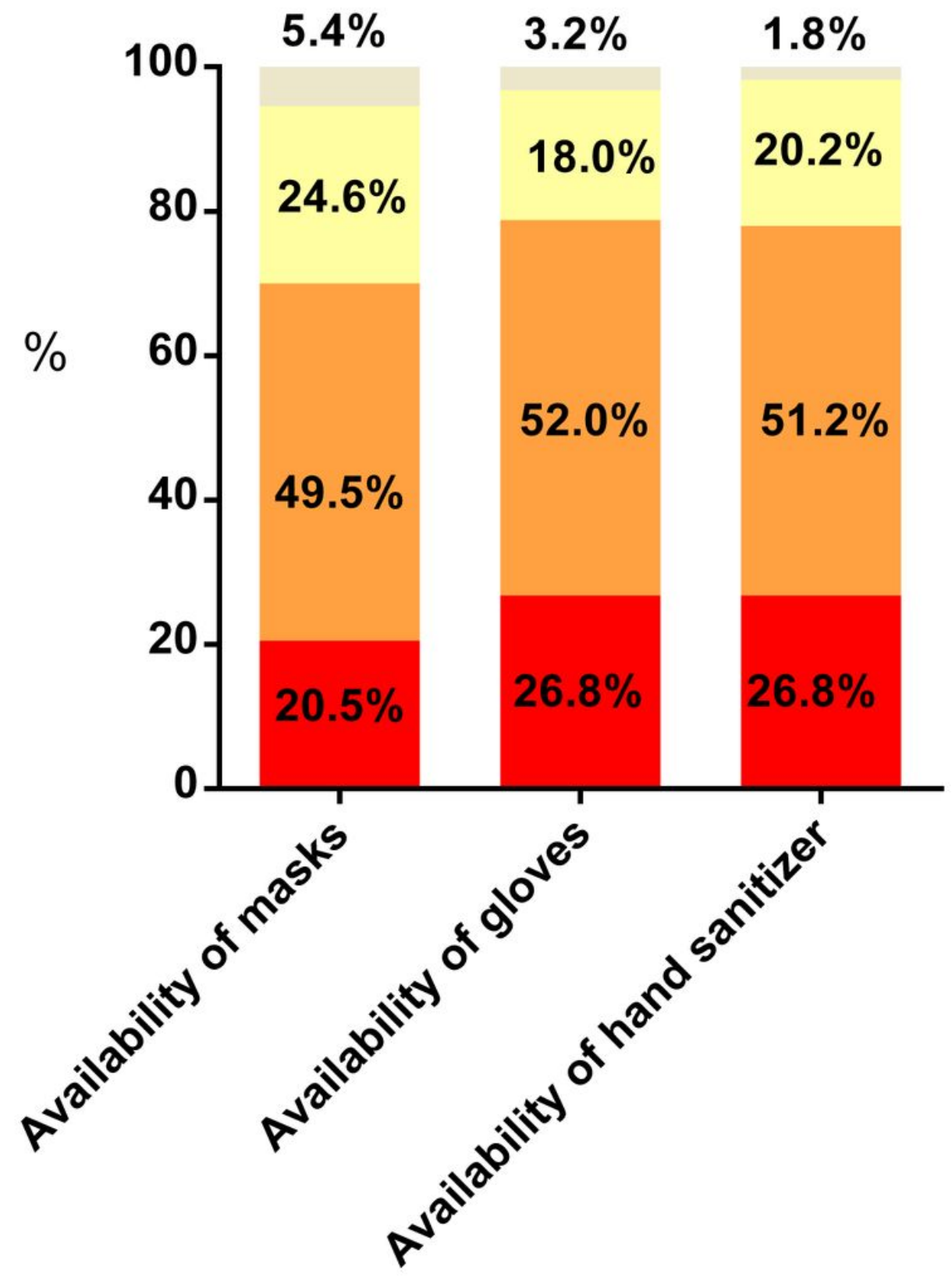

Never Rarely

- Usually

Always

Figure 4

The prevalence of personal protective equipment availability 